\title{
Latest LHCb measurements of semileptonic b-hadron decays
}

\section{Serena Maccolini*}

On behalf of the LHCb Collaboration

University of Bologna and INFN, Bologna, Italy

E-mail: serena.maccolini@cern.ch

\begin{abstract}
Semileptonic $b$-hadron decays proceed via charged-current interactions and provide powerful probes for testing the Standard Model of particle physics and for searching for New Physics effects. The large branching ratios, coupled with excellent particle identification capability and accurate reconstruction of decay vertices, enable the LHCb experiment to perform high-precision measurements of many key quantities, such as CKM matrix elements, $c$ - and $b$-hadron properties, and Lepton Universality. In this contribution, recent results, essential for testing Lepton Universality and understanding hadronic effects, are presented.
\end{abstract}

XXIX International Symposium on Lepton Photon Interactions at High Energies - LeptonPhoton2019 August 5-10, 2019

Toronto, Canada

${ }^{*}$ Speaker. 


\section{Measurement of charmed-baryons lifetimes}

The study of the lifetimes of heavy hadrons containing a $b$ or a $c$ quark allows to test Heavy Quark Expansion (HQE) [1], which can be used to calculate the decay widths of hadrons containing heavy quarks, $Q$, through an expansion in inverse powers of the heavy quark mass, $m_{Q}$. Measurements of charm-hadron lifetimes are sensitive to the higher-order terms in the expansion.

LHCb has recently measured the lifetimes of charmed baryons using inclusive semileptonic $H_{b} \rightarrow H_{c} \mu^{-} X$ decays collected during Run 1 [2], where $H_{b}$ represents a $\Lambda_{b}^{0}, \Xi_{b}^{0}$ or $\Xi_{b}^{-}$baryon and $H_{c}$ corresponds to a $\Lambda_{c}^{+}, \Xi_{c}^{+}$or $\Xi_{c}^{0}$ baryon, respectively. The charmed baryons are reconstructed as $\Lambda_{c}^{+}$ $\rightarrow p K^{-} \pi^{+}, \Xi_{c}^{+} \rightarrow p K^{-} \pi^{+}$and $\Xi_{c}^{0} \rightarrow p K^{-} K^{+} \pi^{+}$. The technique employed to measure the charmbaryon lifetimes follows that used to measure the $\Omega_{b}^{-}$lifetime in Ref. [3]. To reduce systematic uncertainties, the lifetime ratios $r_{H_{c}} \equiv \tau_{H_{c}} / \tau_{D^{+}}$are measured, where the $D^{+}$meson is detected in inclusive semileptonic $B \rightarrow D^{+} \mu^{-} X$ decays with $D^{+} \rightarrow K^{-} \pi^{+} \pi^{+}$.

Each $r_{H_{c}}$ is determined from simultaneous fits to the $H_{c}$ decay-time spectrum and to that of the $D^{+}$meson. Multiplying these ratios by the $D^{+}$lifetime [4], leads to

$$
\begin{aligned}
& \tau_{\Lambda_{c}^{+}}=203.5 \pm 1.0 \pm 1.3 \pm 1.4 \mathrm{fs}, \\
& \tau_{\Xi_{c}^{+}}=456.8 \pm 3.5 \pm 2.9 \pm 3.1 \mathrm{fs}, \\
& \tau_{\Xi_{c}^{0}}=154.5 \pm 1.7 \pm 1.6 \pm 1.0 \mathrm{fs},
\end{aligned}
$$

where the first uncertainty is statistical, the second is systematic and the last uncertainty is due to the uncertainty in the $D^{+}$lifetime. The $\Lambda_{c}^{+}$and $\Xi_{c}^{+}$lifetimes are consistent with the existing world averages. The $\Xi_{c}^{0}$ lifetime is 3.3 standard deviations larger than the world average value of $112_{-10}^{+13}$ fs [4]. These measurements have uncertainties that are approximately 3-4 times smaller than those of the existing world average values, and have a precision comparable to that achieved for charm mesons.

\section{Measurement of $b$-hadron fractions in $13 \mathrm{TeV} p p$ collisions}

Knowledge of the fragmentation fractions of $\bar{B}_{s}^{0}\left(f_{s}\right)$ and $\Lambda_{b}^{0}\left(f_{\Lambda_{b}^{0}}\right)$ hadrons is essential for determining absolute branching ratios $(\mathscr{B})$ of decays of these hadrons at the LHC, allowing measurements, for example, of $\mathscr{B}\left(\bar{B}_{s}^{0} \rightarrow \mu^{+} \mu^{-}\right)$[5] and the future evaluation of $\left|V_{c b}\right|$ from $\Lambda_{b}^{0} \rightarrow \Lambda_{c}^{+} \mu^{-} \bar{v}_{\mu}$ decays [6]. Once these fractions are determined, measurements of absolute branching ratios of $B^{-}$and $\bar{B}^{0}$ mesons performed at $e^{+} e^{-}$colliders operating at the $\Upsilon(4 S)$ resonance can be used to determine the $\bar{B}_{s}^{0}$ and $\Lambda_{b}^{0}$ branching ratios [7].

Recently, LHCb measured the ratios $f_{s} /\left(f_{u}+f_{d}\right)$ and $f_{\Lambda_{b}^{0}} /\left(f_{u}+f_{d}\right)$, where the denominator is the sum of the $B^{-}$and $\bar{B}^{0}$ contributions, in its pseudorapidity $2<\eta<5$ and transverse momentum $4<p_{\mathrm{T}}<25 \mathrm{GeV} / c$, in $13 \mathrm{TeV} p p$ collisions [8]. These ratios can depend on $p_{\mathrm{T}}$ and $\eta$; therefore, the analysis is performed in bins of these two variables. The analysis considers inclusive semileptonic decays $H_{b} \rightarrow H_{c} \mu^{-} X$, where $H_{b}$ indicates a $b$ hadron and $H_{c}$ a charm hadron. Each of the different $H_{c} \mu$ final states can originate from the decay of different $b$ hadrons. The decays accounted for and the detailed equations relating their yields to the final results are given in Ref. [8]. The theoretical basis for this measurement is the near equality of semileptonic decay widths, $\Gamma_{\mathrm{SL}}$, for all $b$-hadron species [9] whose differences are predicted with precisions of about $1 \%$. 
The ratios $f_{s} /\left(f_{u}+f_{d}\right)$ and $f_{\Lambda_{b}^{0}} /\left(f_{u}+f_{d}\right)$ indicate a dependence on $p_{\mathrm{T}}\left(H_{b}\right)$ as shown in Fig. 1 while no dependence on $\eta$ is observed. The integrated values of the ratios are

$$
\begin{aligned}
& \frac{f_{s}}{f_{u}+f_{d}}=0.122 \pm 0.006 \\
& \frac{f_{\Lambda_{b}^{0}}}{f_{u}+f_{d}}=0.259 \pm 0.018
\end{aligned}
$$

where the uncertainties contain both statistical and systematic components, with the latter being dominant.

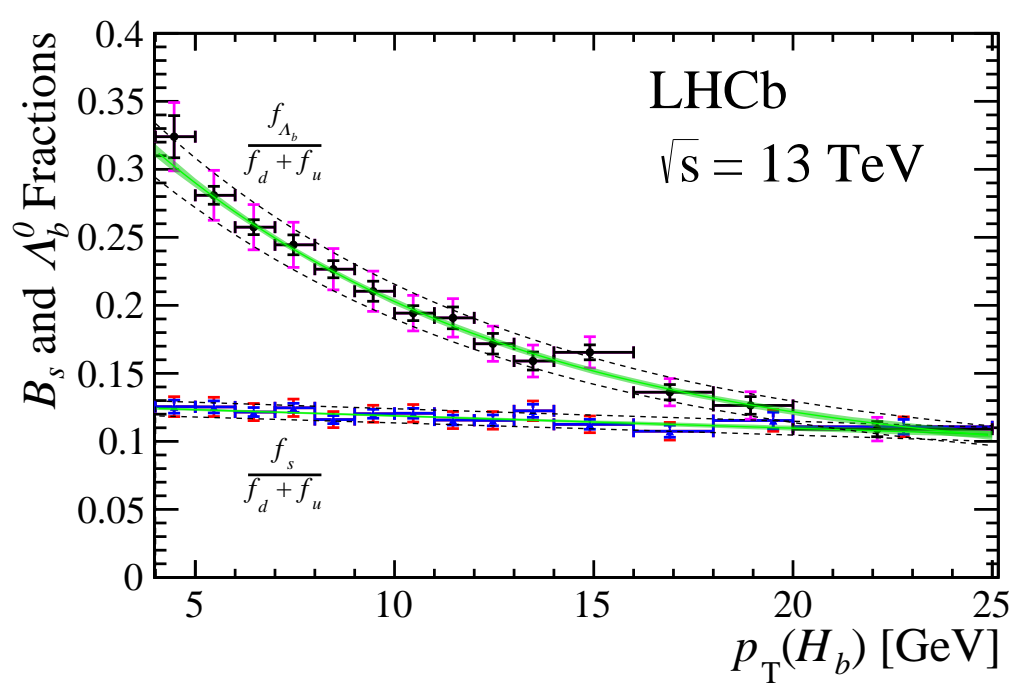

Figure 1: The ratios $f_{s} /\left(f_{u}+f_{d}\right)$ and $f_{\Lambda_{b}^{0}} /\left(f_{u}+f_{d}\right)$ in bins of $p_{\mathrm{T}}\left(H_{b}\right)$. Figure reproduced from Ref. [8].

\section{Search for the rare decay $B^{+} \rightarrow \mu^{+} \mu^{-} \mu^{+} v_{\mu}$}

Purely leptonic decays of the $B^{+}$meson are rare, as branching ratios are proportional to the squared magnitude of the Cabibbo-Kobayashi-Maskawa (CKM) matrix element $V_{u b}$. Among these processes, the $B^{+} \rightarrow \mu^{+} v_{\mu}$ decay has a precise Standard Model (SM) prediction [10] given the absence of hadrons in the final state. Due to helicity suppression, they are also highly sensitive to particles predicted in extensions of the SM. The radiative version of the muonic decay, $B^{+} \rightarrow \mu^{+} v_{\mu} \gamma$, is important for two reasons; it is a background for the $B^{+} \rightarrow \mu^{+} v_{\mu}$ decay, and its branching ratio is a direct measurement of the inverse moment of the $B$ meson light cone distribution amplitude, which is very difficult to calculate theoretically [11].

A $B$ decay vertex with just a single charged particle makes a search for the $B^{+} \rightarrow \mu^{+} v_{\mu}$ and $B^{+} \rightarrow \mu^{+} v_{\mu} \gamma$ decays highly challenging in the LHC environment. This problem is not present for the decay $B^{+} \rightarrow \mu^{+} \mu^{-} \mu^{+} v_{\mu}$. The decay receives a contribution from the $B^{+} \rightarrow \mu^{+} v_{\mu} \gamma^{*}$ decay with $\gamma^{*} \rightarrow \mu^{+} \mu^{-}$, where the annihilation to the $\mu^{+} v_{\mu}$ pair occurs through an intermediate $B^{*}$ meson. It also receives contributions from the $B^{+} \rightarrow \mu^{+} v_{\mu} V$ amplitude, where $V$ denotes a vector meson such as the $\omega$ or the $\rho$, that can decay to a pair of muons. With these contributions, nearly 
all decays have a muon pair with a mass below $1 \mathrm{GeV} / c^{2}$. A recent theoretical calculation based on vector-meson dominance predicts that the corresponding branching ratio, $\mathscr{B}\left(B^{+} \rightarrow \mu^{+} \mu^{-} \mu^{+} v_{\mu}\right)$, is around $1.3 \times 10^{-7}[12]$.

A search for the decay $B^{+} \rightarrow \mu^{+} \mu^{-} \mu^{+} v_{\mu}$ has been performed by the LHCb experiment using $p p$ collision data corresponding to an integrated luminosity of $4.7 \mathrm{fb}^{-1}$ collected during the years 2011, 2012 and 2016 [13]. This search uses a partial reconstruction method that infers the momentum of the missing neutrino to obtain a mass estimate of $B^{+} \rightarrow \mu^{+} \mu^{-} \mu^{+} v_{\mu}$ decays. The distribution of this variable is used to perform a fit to obtain the signal yield. The determined signal yields is $-25 \pm 16$, resulting in a limit on the branching ratio of $\mathscr{B}\left(B^{+} \rightarrow \mu^{+} \mu^{-} \mu^{+} v_{\mu}\right)<1.6 \times 10^{-8}$ at $95 \%$ confidence level. This limit is in tension with a theoretical calculation based on the vectordominance model [12].

\section{References}

[1] I. I. Bigi, N. G. Uraltsev and A. I. Vainshtein, Nonperturbative corrections to inclusive beauty and charm decays. QCD versus phenomenological models, Phys.Lett. B293 (1992) 430 [hep-ph/9207214].

[2] LHCb collaboration, Precision measurement of the $\Lambda_{c}^{+}, \Xi_{c}^{+}$, and $\Xi_{c}^{0}$ baryon lifetimes, Phys. Rev. D100 (2019) 032001 LHCb-PAPER-2019-008 CERN-EP-2019-122, [1906 . 08350].

[3] LHCb collaboration, Measurements of the mass and lifetime of the $\Omega_{b}^{-}$baryon, Phys. Rev. D93 (2016) 092007 LHCb-PAPER-2016-008, CERN-EP-2016-081, [1604 . 01412].

[4] Particle Data Group collaboration, Review of particle physics, Phys. Rev. D98 (2018) 030001.

[5] CMS, LHCb collaboration, Observation of the rare $B_{s}^{0} \rightarrow \mu^{+} \mu^{-}$decay from the combined analysis of CMS and LHCb data, Nature 522 (2015) 68 [1411.4413].

[6] LHC $b$ collaboration, Measurement of the shape of the $\Lambda_{b}^{0} \rightarrow \Lambda_{c}^{+} \mu^{-} \bar{v}_{\mu}$ differential decay rate, Phys. Rev. D96 (2017) 112005 LHCb-PAPER-2017-016, CERN-EP-2017-164, [170 9 . 01920].

[7] Particle Data Group collaboration, Review of Particle Physics, Phys. Rev. D98 (2018) 030001.

[8] LHCb collaboration, Measurement of b-hadron fractions in 13 TeV pp collisions, Phys. Rev. D101 (2019) 031102(R) LHCb-PAPER-2018-050 CERN-EP-2019-016, [1902 . 06794].

[9] I. I. Bigi, T. Mannel and N. Uraltsev, Semileptonic width ratios among beauty hadrons, JHEP 09 (2011) 012 [1105.4574].

[10] D. Silverman and H. Yao, Relativistic Treatment of Light Quarks in D and B Mesons and W Exchange Weak Decays, Phys. Rev. D38 (1988) 214.

[11] M. Beneke and J. Rohrwild, B meson distribution amplitude from $B \rightarrow \gamma l v$, Eur. Phys. J. C71 (2011) 1818 [1110.3228].

[12] A. V. Danilina and N. V. Nikitin, Four-Leptonic Decays of Charged and Neutral B Mesons within the Standard Model, Phys. Atom. Nucl. 81 (2018) 347.

[13] LHC $b$ collaboration, Search for the rare decay $B^{+} \rightarrow \mu^{+} \mu^{-} \mu^{+} v_{\mu}$, Eur. Phys. J. C79 (2019) 675 LHCb-PAPER-2018-037 CERN-EP-2018-293, [1812.06004]. 